\title{
Theory of integer equivariant estimation with application to GNSS
}

\author{
P. J. G. Teunissen \\ Department of Mathematical Geodesy and Positioning, Delft University of Technology, Thijsseweg 11, 2629 JA Delft, \\ The Netherlands; e-mail: p.j.g.teunissen@citg.tudelft.nl; Tel.: +31-15-278-2558; Fax: + 31-15-278-3711
}

Received: 18 November 2002 / Accepted: 3 June 2003

\begin{abstract}
Carrier phase ambiguity resolution is the key to high-precision global navigation satellite system (GNSS) positioning and navigation. It applies to a great variety of current and future models of GPS, modernized GPS and Galileo. The so-called 'fixed' baseline estimator is known to be superior to its 'float' counterpart in the sense that its probability of being close to the unknown but true baseline is larger than that of the 'float' baseline, provided that the ambiguity success rate is sufficiently close to its maximum value of one. Although this is a strong result, the necessary condition on the success rate does not make it hold for all measurement scenarios. It is discussed whether or not it is possible to take advantage of the integer nature of the ambiguities so as to come up with a baseline estimator that is always superior to both its 'float' and its 'fixed' counterparts. It is shown that this is indeed possible, be it that the result comes at the price of having to use a weaker performance criterion. The main result of this work is a GaussMarkov-like theorem which introduces a new minimum variance unbiased estimator that is always superior to the well-known best linear unbiased (BLU) estimator of the Gauss-Markov theorem. This result is made possible by introducing a new class of estimators. This class of integer equivariant estimators obeys the integer remove-restore principle and is shown to be larger than the class of integer estimators as well as larger than the class of linear unbiased estimators. The minimum variance unbiased estimator within this larger class is referred to as the best integer equivariant (BIE) estimator. The theory presented applies to any model of observation equations having both integer and real-valued parameters, as well as for any probability density function the data might have.
\end{abstract}

Keywords: Global navigation satellite system ambiguity resolution - Integer equivariant estimation - Minimum variance unbiased estimation

\section{Introduction}

Global navigation satellite system (GNSS) ambiguity resolution is the process of resolving the unknown cycle ambiguities of double-difference (DD) carrier phase data. Its practical importance becomes clear when we realize the great variety of current and future GNSS models to which it applies. These models may differ greatly in complexity and diversity. They range from single-baseline models used for kinematic positioning to multi-baseline models used as a tool for studying geodynamic phenomena. The models may or may not have the relative receiver-satellite geometry included. They may also be discriminated as to whether the slave receiver(s) is stationary or in motion. When in motion, we solve for one or more trajectories, since with the receiver-satellite geometry included, we will have new coordinate unknowns for each epoch. We may also discriminate between the models as to whether or not the differential atmospheric delays (ionosphere and troposhere) are included as unknowns. In the case of sufficiently short baselines they are usually excluded.

Apart from the current global positioning system (GPS) models, carrier phase ambiguity resolution also applies to the future modernized GPS and the future European Galileo GNSS. An overview of GNSS models, together with their applications in surveying, navigation, geodesy and geophysics, can be found in textbooks such as Leick (1995), Parkinson and Spilker (1996), Strang and Borre (1997), Teunissen and Kleusberg (1998), Hofmann-Wellenhof et al. (2001) and Misra and Enge (2001).

Since carrier phase ambiguity resolution is the key to high-precision GNSS positioning and navigation, the availability of a theory of integer inference is a prerequisite for a proper handling and understanding of the various intricate aspects of ambiguity resolution. The usual approach to carrier phase ambiguity resolution is to resolve the ambiguities once the probability of correct 
integer estimation, the ambiguity success rate, is sufficiently close to one. When this happens we can show that the so-called 'fixed' baseline estimator is superior to its 'float' counterpart in the sense that its probability of being close to the unknown but true baseline is larger than that of the 'float' baseline. Although this is a strong result, the down side of it is that it only holds true when the success rate is sufficiently large. It is this observation that formed the basis of our motivation for conducting the present study. The question that will be answered is whether or not it is possible to take advantage of the integer nature of the ambiguities so as to come up with a baseline estimator that is always superior to both its 'float' and its 'fixed' counterparts. We will show that this is indeed possible, be it that the result comes at the price of having to use a weaker performance criterion. The performance criterion chosen is the mean square error (MSE). The reason for taking the MSE as the weaker performance criterion is twofold. First, it is a wellknown probabilistic criterion for measuring the closeness of an estimator to its target value. Second, this criterion is also often used as measure for the quality of the 'float' solution itself.

Although the present study was motivated by the problem of GNSS ambiguity resolution, the theory that will be developed is of interest in its own right. It applies to any model of observation equations having unknown integer parameters as well as unknown real-valued parameters. Our main result is a Gauss-Markov-like theorem which introduces an estimator that is always superior to the well-known best linear unbiased (BLU) estimator of the Gauss-Markov theorem. The GaussMarkov theorem states that the minimum variance unbiased estimator within the class of linear estimators is given by the least-squares (LS) estimator. Our theorem states that the minimum variance unbiased estimator within the class of integer equivariant (IE) estimators is given by the least mean-squared (LMS) estimator. This estimator, referred to as the best integer equivariant (BIE) estimator, is superior to the BLU estimator since the class of linear unbiased estimators can be shown to be a subset of the class of IE estimators. In the same sense it can also be shown to be superior to integer estimators.

This contribution is organized as follows. In Sect. 2 we give a brief review of the present theory of integer estimation. This includes the definition of the class of admissible integer estimators. This class is taken as our point of departure for introducing the class of integer equivariant (IE) estimators in Sect. 3. Although this new class is larger than the class of integer estimators, it has been chosen such that its members still obey the integer remove-restore principle. When estimating ambiguities in case of GNSS, for instance, it seems reasonable to require, when adding an arbitrary number of cycles to the carrier phase data, that the solution of the integer ambiguities gets shifted by the same integer amount. In Sect. 3 we show that the class of linear unbiased estimators is a subset of the class of IE estimators. We also give a useful representation of IE estimators. This representation reveals the structure of IE estimators and easily allows us to devise our own IE estimator.
In Sect. 4 we use the MSE criterion to find the best estimator within the IE class for any linear function of both the integer as well as real-valued parameters of the general GNSS model. We give an explicit expression for the BIE estimator. Although IE estimators are not unbiased in general, we show in Sect. 5 that the BIE estimator is unbiased. This implies that the BIE estimator is identical to the BIE unbiased estimator. This result gives rise to our Gauss-Markov-like theorem stating the minimum variance unbiasedness property of the BIE estimator. Although the BIE estimator holds true for any probability density function the data might have, we also consider the special case of normally distributed data. For this special case it is shown that the BIE estimator of the baseline can be obtained in a way which is very similar to the three-step procedure of current methods of ambiguity resolution, the only difference being that the integer ambiguity estimator needs to be replaced by its BIE counterpart.

\section{Integer estimation}

\subsection{The GNSS model}

As our point of departure we take the following system of linear observation equations:

$E\{y\}=A a+B b, \quad a \in Z^{n}, \quad b \in R^{p}$

with $E\{\cdot\}$ the mathematical expectation operator, $y$ the $m$ vector of observables, $a$ the $n$ vector of unknown integer parameters and $b$ the $p$ vector of unknown realvalued parameters. The $m \times(n+p)$ design matrix $(A, B)$ is assumed to be of full rank.

All the linear(ized) GNSS models can in principle be cast in the above frame of observation equations. The data vector $y$ will then usually consist of the 'observed minus computed' single- or dual-frequency DD phase and/or pseudorange (code) observations accumulated over all observation epochs. The entries of vector $a$ are then the DD carrier phase ambiguities, expressed in units of cycles rather than range, while the entries of the vector $b$ will consist of the remaining unknown parameters, such as for instance baseline components (coordinates) and possibly atmospheric delay parameters (troposphere, ionosphere).

Although the theory that will be developed in this contribution holds true for any application for which the observation equations can be formulated as Eq. (1), we will still refer to it as the GNSS model.

\subsection{The three-step solution}

The procedure which is usually followed for solving the GNSS model can be divided into three steps. In the first step we simply discard the integer constraints $a \in Z^{n}$ and perform a standard LS adjustment. As a result we obtain the LS estimators of $a$ and $b$ as 
$\hat{a}=\left(\bar{A}^{T} Q_{y}^{-1} \bar{A}\right)^{-1} \bar{A}^{T} Q_{y}^{-1} y$

$\hat{b}=\left(\bar{B}^{T} Q_{y}^{-1} \bar{B}\right)^{-1} \bar{B}^{T} Q_{y}^{-1} y$

with $Q_{y}$ the vc matrix of the observables, $\bar{A}=P_{B}^{\perp} A$, $\bar{B}=P_{A}^{\perp} B$, and the two orthogonal projectors $P_{B}^{\perp}=$ $I_{m}-B\left(B^{T} Q_{y}^{-1} B\right)^{-1} B^{T} Q_{y}^{-1}$ and $P_{A}^{\perp}=I_{m}-A\left(A^{T} Q_{y}^{-1} A\right)^{-1}$. $A^{T} Q_{y}^{-1}$. This solution is usually referred to as the 'float' solution.

In the second step the 'float' estimator $\hat{a}$ is used to compute the corresponding integer estimator $\check{a} \in Z^{n}$. This implies that a mapping $S$ from the $n$-dimensional space of reals to the $n$-dimensional space of integers is introduced such that

$\check{a}=S(\hat{a}), \quad S: R^{n} \mapsto Z^{n}$

This integer estimator is then used in the third and final step to adjust the 'float' estimator $\hat{b}$. As a result we obtain the so-called 'fixed' estimator of $b$ as

$\check{b}=\hat{b}-Q_{\hat{b} \hat{a}} Q_{\hat{a}}^{-1}(\hat{a}-\check{a})$

in which $Q_{\hat{a}}$ denotes the vc matrix of $\hat{a}$ and $Q_{\hat{b} \hat{a}}$ denotes the covariance matrix of $\hat{b}$ and $\hat{a}$. This 'fixed' estimator can alternatively be expressed as $\check{b}=$ $\left(B^{T} Q_{y}^{-1} B\right)^{-1} B^{T} Q_{y}^{-1}(y-A \check{a})$. Note that only two of the three steps are needed if we are only interested in obtaining an integer solution for $a$. In the case of GNSS, however, we are particularly interested in the solution of the third step as it contains the solution for the baseline coordinates. All three steps are therefore needed in the case of GNSS. In the following we will use the terminology of GNSS and refer to $\hat{b}$ and $\breve{b}$ as, respectively, the 'float' and 'fixed' baseline estimators.

The above three-step procedure is still ambiguous in the sense that it leaves room for choosing the integer map $S$. Different choices for $S$ will lead to different integer estimators $\check{a}$ and thus also to different baseline estimators $\breve{b}$. We can therefore now think of constructing integer maps which possess certain desirable properties.

\subsection{A class of integer estimators}

It will be clear that the map $S$ will not be one-to-one due to the discrete nature of $Z^{n}$. Instead it will be a manyto-one map. This implies that different real-valued vectors will be mapped to one and the same integer vector. We can therefore assign a subset $S_{z} \subset R^{n}$ to each integer vector $z \in Z^{n}$

$S_{z}=\left\{x \in R^{n} \mid z=S(x)\right\}, \quad z \in Z^{n}$

The subset $S_{z}$ contains all real-valued vectors that will be mapped by $S$ to the same integer vector $z \in Z^{n}$. This subset is referred to as the pull-in region of $z$. It is the region in which all vectors are pulled to the same integer vector $z$.

Since the pull-in regions define the integer estimator completely, we can define classes of integer estimators by imposing various conditions on the pull-in regions. One such class was introduced by Teunissen (1999a) and is referred to as the class of admissible integer estimators.

Definition 1: admissible integer estimators. The integer estimator $\check{a}=S(\hat{a})$ is said to be admissible if its pull-in regions satisfy

1. $\bigcup_{z \in Z^{n}} S_{z}=R^{n}$

2. $\operatorname{Int}\left(S_{z_{1}}\right) \bigcap \operatorname{Int}\left(S_{z_{2}}\right)=\emptyset, \forall z_{1}, z_{2} \in Z^{n}, z_{1} \neq z_{2}$

3. $S_{z}=z+S_{0}, \forall z \in Z^{n}$

This definition is motivated as follows. Each one of the above three conditions describes a property of which it seems reasonable is possessed by an arbitrary integer estimator. The first condition states that the pull-in regions should not leave any gaps and the second that they should not overlap. The absence of gaps is needed in order to be able to map any 'float' solution $\hat{a} \in R^{n}$ to $Z^{n}$, while the absence of overlaps is needed to guarantee that the 'float' solution is mapped to just one integer vector. Note that we allow the pull-in regions to have common boundaries. This is permitted if we assume to have zero probability that $\hat{a}$ lies on one of the boundaries. This will be the case when the probability density function (PDF) of $\hat{a}$ is continuous.

The third and last condition of the definition follows from the requirement that $S(x+z)=S(x)+z$, $\forall x \in R^{n}, z \in Z^{n}$. This condition is also a reasonable one to ask for. It states that when the 'float' solution $\hat{a}$ is perturbed by $z \in Z^{n}$, the corresponding integer solution is perturbed by the same amount. This property allows us to apply the integer remove-restore technique: $S(\hat{a}-z)+z=S(\hat{a})$. It therefore allows us to work with the fractional parts of the entries of $\hat{a}$, instead of with its complete entries.

Using the pull-in regions, we can give an explicit expression for the corresponding integer estimator $\breve{a}$. It reads

$\check{a}=\sum_{z \in Z^{n}} z s_{z}(\hat{a})$

with the indicator function $s_{z}(\hat{a})=1$ if $\hat{a} \in S_{z}$ and $s_{z}(\hat{a})=0$ otherwise. Note that the $s_{z}(\hat{a})$ can be interpreted as weights, since $\sum_{z \in Z^{n}} s_{z}(\hat{a})=1$. The integer estimator $\check{a}$ is therefore equal to a weighted sum of integer vectors with binary weights.

\subsection{The performance of the baseline estimators}

As mentioned earlier the 'fixed' baseline estimator $\check{b}$ depends on the chosen integer estimator $\check{a}$. We may also write $\check{b}$ as

$\check{b}=\hat{b}(a)+Q_{\hat{b} \hat{a}} Q_{\hat{a}}^{-1}(\check{a}-a)$

with the conditional baseline estimator $\hat{b}(a)=\hat{b}-$ $Q_{\hat{b} \hat{a}} Q_{\hat{a}}^{-1}(\hat{a}-a)$. Since $a$ is assumed known in the case of $\hat{b}(a)$, the conditional baseline estimator is the best possible estimator of $b$. It is unbiased, it has a precision which is better than that of $\hat{b}$, and in the case that $y$ is normally distributed its PDF is also more peaked than 
that of the 'float' estimator. The best we can hope for in the case of $\check{b}$ is therefore that $\check{a}=a$. However, this requires that the probability of correct integer estimation, $P(\check{a}=a)$, equals one. Hence, of all admissible integer estimators the preferred estimator is the one that maximizes the probability of correct integer estimation. It was shown by Teunissen (1999b) that in the case of elliptically contoured distributions, the integer least-squares (ILS) estimator is the preferred estimator. Thus, if $\check{a}_{\mathrm{ILS}}=\arg \min _{z \in Z^{n}}(\hat{a}-z)^{T} Q_{\hat{a}}^{-1}(\hat{a}-z)$, then $P\left(\check{a}_{\mathrm{ILS}}=a\right) \geq P(\check{a}=a)$ for any admissible estimator $\check{a}$. And once the probability of correct integer estimation is sufficiently close to one, we have

$P\left(\check{b} \in E_{b}\right) \geq P\left(\hat{b} \in E_{b}\right)$

for any convex region $E_{b} \subset R^{p}$ symmetric with respect to $b$. The usual approach taken with GNSS is therefore to use the ILS estimator for computing the 'fixed' baseline estimator, once it has been verified that $P\left(\check{a}_{\mathrm{ILS}}=a\right)$ is sufficiently close to one, see (Teunissen 1993, 1995).

When valid, the inequality of Eq. (8) is a very strong result. It states that the 'fixed' baseline estimator $\check{b}$ has a higher probability of being close to $b$ than its 'float' counterpart. The down side of the inequality is, however, that it is ony valid if $P(\check{a}=a)$ is sufficiently close to one. This being the case, we may wonder whether it would not be possible to devise a baseline estimator which always outperforms its 'float' counterpart. Such an approach can, however, only be successful if we use a weaker performance criterion than that of Eq. (8). Furthermore, assuming that we will be successful in finding an optimal estimator using this weaker criterion, then this new estimator will always be better than its 'float' counterpart only if we consider a class of estimators which encompasses the class of estimators in which the 'float' estimator resides. This means that, as a start, we should at least consider a class of estimators which is larger than the above considered class of integer estimators.

\section{Integer equivariant estimation}

\subsection{Class of IE estimators}

We will now introduce a new class of estimators which is larger than the previously defined class of integer estimators. In order to be general enough, we consider estimating an arbitrary linear function of the two types of unknown parameters of the GNSS model of Eq. (1)

$\theta=l_{a}^{T} a+l_{b}^{T} b, \quad l_{a} \in R^{n}, \quad l_{b} \in R^{p}$

Thus if $l_{b}=0$ then linear functions of the ambiguities are estimated, whereas if $l_{a}=0$ then linear functions of the baseline are estimated. Linear functions of both the ambiguities and the baseline, such as carrier phases, are estimated in the case that $l_{a} \neq 0$ and $l_{b} \neq 0$.

It seems reasonable that the estimator should at least obey the integer remove-restore principle. When estimating ambiguities in the case of GNSS, for instance, when adding an arbitrary number of cycles to the carrier phase data, we would like the solution of the integer ambiguities to be shifted by the same integer amount. For the estimator of $\theta$ this would mean that adding $A z$ to $y$, with arbitrary $z \in Z^{n}$, must result in a shift of $l_{a}^{T} z$. Likewise, it seems reasonable to require of the estimator that adding $B \zeta$ to $y$, with arbitrary $\zeta \in R^{p}$, results in a shift of $l_{b}^{T} \zeta$. After all, we would not like the integer part of the estimator to become contaminated by such an addition to $y$. Estimators of $\theta$ that fulfil these two conditions will be called integer equivariant (IE). Hence, they are defined as follows.

Definition 2: integer equivariant (IE) estimators. The estimator $\hat{\theta}_{I E}=f_{\theta}(y)$, with $f_{\theta}: R^{m} \mapsto R$, is said to be an IE estimator of $\theta=l_{a}^{T} a+l_{b}^{T} b$ if

$\begin{array}{ll}f_{\theta}(y+A z)=f_{\theta}(y)+l_{a}^{T} z, & \forall y \in R^{m}, \quad z \in Z^{n} \\ f_{\theta}(y+B \zeta)=f_{\theta}(y)+l_{b}^{T} \zeta, & \forall y \in R^{m}, \quad \zeta \in R^{p}\end{array}$

It is not difficult to verify that the integer estimators of the previous section are IE. Simply check that the above two conditions are indeed fulfilled by the estimator $\check{\theta}=l_{a}^{T} \check{a}+l_{b}^{T} \check{b}$. The converse, however, is not necessarily true. The class of IE estimators is therefore a larger class.

We will now show that the class of IE estimators is also larger than the class of linear unbiased estimators. Let $f_{\theta}^{T} y$, for some $f_{\theta} \in R^{m}$, be the linear estimator of $\theta=l_{a}^{T} a+l_{b}^{T} b$. For it to be unbiased we require, using $E\{y\}=A a+B b, \quad$ that $f_{\theta}^{T} A a+f_{\theta}^{T} B b=l_{a}^{T} a+l_{b}^{T} b$, $\forall a \in R^{n}, b \in R^{p}$ holds true, or that both $l_{a}=A^{T} f_{\theta}$ and $l_{b}=B^{T} f_{\theta}$ hold true. But this is equivalent to stating that $f_{\theta}^{T}(y+A a)=f_{\theta}^{T} y+l_{a}^{T} a, \quad \forall y \in R^{m}, a \in R^{n}$ $f_{\theta}^{T}(y+B b)=f_{\theta}^{T} y+l_{b}^{T} b, \quad \forall y \in R^{m}, \quad b \in R^{p}$

Comparing this result with Eq. (10) shows that the condition of linear unbiasedness is more restrictive than the condition of integer equivariance. Hence, the class of linear unbiased estimators is a subset of the class of IE estimators. This result also automatically implies that IE estimators exist which are unbiased. Thus, if we denote the class of IE estimators as IE, the class of unbiased estimators as $\mathrm{U}$, the class of unbiased IE estimators as $I E U$, the class of unbiased integer estimators as IU, and the class of linear unbiased estimators as LU, we may summarize their relationships as: IEU $=\mathrm{IE} \cap \mathrm{U} \neq \emptyset$, LU $\subset$ IEU and IU $\subset$ IEU (see Fig. 1).

\subsection{Representation of IE estimators}

In order to obtain a better understanding of how IE estimators operate, it would be useful to have a representation that reveals their structure. One such representation is given in the following lemma.

Lemma 1: IE-representation. Let $\hat{\theta}_{\mathrm{IE}}=f_{\theta}(y)$ be the IEestimator of $\theta=l_{a}^{T} a+l_{b}^{T} b$, let $y=A \alpha+B \beta+C \gamma$, with the $m \times(m-n-p)$ matrix $C$ chosen such that $(A, B, C)$ is invertible, and let $g_{\theta}(\alpha, \beta, \gamma)=f_{\theta}(A \alpha+B \beta+C \gamma)$. Then functions $h_{\theta}: R^{n} \times R^{m-n-p_{\longmapsto}}$ exist such that 


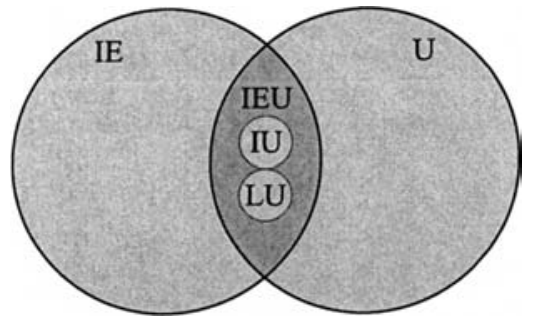

Fig. 1. The set relationships between the different classes of estimators: integer equivariant estimators IE, unbiased estimators $\mathrm{U}$, unbiased integer equivariant estimators IEU, unbiased integer estimators IU, and linear unbiased estimators LU

$g_{\theta}(\alpha, \beta, \gamma)=l_{a}^{T} \alpha+l_{b}^{T} \beta+h_{\theta}(\alpha, \gamma)$

with $h_{\theta}(\alpha+z, \gamma)=h_{\theta}(\alpha, \gamma)$ for all $z \in Z^{n}$.

Proof of Lemma 1 is given in the appendix.

This representation turns out to be useful in some of the later proofs. Also note that Lemma 1 now easily allows us to design our own IE estimator. When devising our own IE estimator, there are essentially two types of degrees of freedom involved: the choice of the matrix $C$ and the choice of the function $h_{\theta}$.

The following are some examples of IE estimators obtained for specific choices of $C$ and $h_{\theta}$.

Example 1. For arbitrary $C$ and $h_{\theta}=0$ we obtain

$\hat{\theta}_{\mathrm{IE}}=l_{a}^{T} \alpha+l_{b}^{T} \beta$

Note that this is a linear unbiased estimator of $\theta$ for any choice of $C$. Hence, matrix $C$ governs the choice of these linear unbiased estimators.

Example 2. For $h_{\theta}=0$ and $C$ chosen such that $C^{T} Q_{y}^{-1}(A, B)=0$ we obtain the LS estimator

$\hat{\theta}_{\mathrm{IE}}=l_{a}^{T} \hat{a}+l_{b}^{T} \hat{b}$

Example 3. For $h_{\theta}(\alpha, \gamma)=-\left(l_{a}^{T}+l_{b}^{T} Q_{\hat{b} \hat{a}} Q_{\hat{a}}^{-1}\right)(\alpha-S(\alpha))$ and $C$ chosen such that $C^{T} Q_{y}^{-1}(A, B)=0$ we obtain the estimator

$\hat{\theta}_{\mathrm{IE}}=l_{a}^{T} \check{a}+l_{b}^{T} \check{b}$

Example 4. For $h_{\theta}(\alpha, \gamma)=-\left(l_{a}^{T}+l_{b}^{T} Q_{\hat{b} \hat{a}} Q_{\hat{a}}^{-1}\right)(\alpha-S(\alpha))$, $S(\alpha)=\arg \min _{z \in Z^{n}}(\alpha-z)^{T} Q_{\hat{a}}^{-1}(\alpha-z)$ and $C$ chosen such that $C^{T} Q_{y}^{-1}(A, B)=0$ we obtain the integer LS estimator

$\hat{\theta}_{\mathrm{IE}}=l_{a}^{T} \check{a}_{\mathrm{ILS}}+l_{b}^{T} \check{b}_{\mathrm{ILS}}$

\section{Best integer equivariant estimation}

\subsection{The BIE estimator}

Having defined the class of IE estimators we will now look for an IE estimator which is 'best' in a certain sense. We will denote our best integer equivariant (BIE) estimator of $\theta$ as $\hat{\theta}_{\mathrm{BIE}}$ and use the MSE as our criterion of 'best'. The BIE estimator will therefore be defined as

$\hat{\theta}_{\mathrm{BIE}}=\arg \min _{f_{\theta} \in \mathrm{IE}} E\left\{\left(f_{\theta}(y)-\theta\right)^{2}\right\}$

in which IE stands for the class of IE estimators. The minimization is thus taken over all IE functions that satisfy the conditions of Definition 2.

The reason for choosing the MSE criterion is twofold. First, it is a well-known probabilistic criterion for measuring the closeness of an estimator to its target value, in our case $\theta$. Second, the MSE criterion is also often used as measure for the quality of the 'float' solution itself. The following theorem gives the solution to the above minimization problem of Eq. (13).

Theorem 1: best integer equivariant estimation. Let $y \in R^{m}$ have mean $E\{y\}=A a+B b$ and $p d f p_{y}(y)$, and let $\hat{\theta}_{\mathrm{BIE}}$ be the BIE estimator of $\theta=l_{a}^{T} a+l_{b}^{T} b$. Then

$\hat{\theta}_{\mathrm{BIE}}=\frac{\sum_{z \in Z^{n}} \int_{R^{p}}\left(l_{a}^{T} z+l_{b}^{T} \beta\right) p_{y}(y+A(a-z)+B(b-\beta)) \mathrm{d} \beta}{\sum_{z \in Z^{n}} \int_{R^{p}} p_{y}(y+A(a-z)+B(b-\beta)) \mathrm{d} \beta}$

Proof of Theorem 1 is given in the appendix.

Note that the BIE estimator can also be written as

$\hat{\theta}_{\mathrm{BIE}}=l_{a}^{T} \hat{a}_{\mathrm{BIE}}+l_{b}^{T} \hat{b}_{\mathrm{BIE}}$

with

$\hat{a}_{\mathrm{BIE}}=\sum_{z \in Z^{n}} z w_{z}(y), \quad \sum_{z \in Z^{n}} w_{z}(y)=1$

$\hat{b}_{\mathrm{BIE}}=\int_{R^{p}} \beta w_{\beta}(y) \mathrm{d} \beta, \quad \int_{R^{p}} w_{\beta}(y) \mathrm{d} \beta=1$

in which the weighting functions $w_{z}(y)$ and $w_{\beta}(y)$ are defined by Eq. (14). This shows that the BIE estimator of the integer parameter vector $a$ is also a weighted sum of all integer vectors in $Z^{n}$, just like $\check{a}$ of Eq. (6) is. In the present case, however, the weights are not binary. They vary between 0 and 1 , and their values are determined by $y$ and its PDF. As a consequence the estimator $\hat{a}_{\mathrm{BIE}}$ will in general be real valued, instead of integer valued.

The above theorem holds true for any PDF the vector of observables $y$ might have. This is therefore a very general result indeed. A closer look at Eq. (14) reveals however, that we need $a$ and $b$, and therefore $\theta$, in order to compute $\hat{\theta}_{\mathrm{BIE}}$. The dependence on $a$ and $b$ is present in the numerator of Eq. (14) and not in its denominator. The summation over all integer vectors in $Z^{n}$ and the integration over $R^{p}$ makes the dependence on $a$ and $b$ disappear in the denominator. If the dependence of $\hat{\theta}_{\mathrm{BIE}}$ on $\theta$ persisted we would not be able to compute the BIE estimator. Note, however, that this dependence disappears in the case that the PDF of $y$ has the structure $p_{y}(y)=f(y-A a-B b)$; this property is fortunately still true for a large class of PDFs. 


\subsection{BIE is better than BLU}

A direct and important consequence of the above theorem is that the BIE estimator is always better than or at least as good as any integer estimator as well as any linear unbiased estimator. After all, the class of integer estimators and the class of linear unbiased estimators are both subsets of the class of IE estimators. The BIE estimator is therefore also better than the BLU estimator. The BLU estimator is the minimum variance estimator of the class of linear unbiased estimators and it is given by the wellknown Gauss-Markov theorem. This theorem, when adapted to our GNSS model of Eq. (1), reads as follows.

Gauss-Markov Theorem: minimum variance linear unbiased estimation. Let $y \in R^{m}$ have mean $E\{y\}=A a+B b$ and dispersion $D\{y\}=Q_{y}$, and let $\hat{\theta}_{B L U}$ be the $B L U$ estimator of $\theta=l_{a}^{T} a+l_{b}^{T} b$. Then

$\hat{\theta}_{\mathrm{BLU}}=l_{a}^{T} \hat{a}+l_{b}^{T} \hat{b}$

with $\hat{a}$ and $\hat{b}$ as given by Eq. (2). We therefore have

$\operatorname{MSE}\left(\hat{\theta}_{\mathrm{BIE}}\right) \leq \operatorname{MSE}\left(\hat{\theta}_{\mathrm{BLU}}\right)$

The two estimators $\hat{\theta}_{\mathrm{BIE}}$ and $\hat{\theta}_{\mathrm{BLU}}$ both minimize the MSE within their class. In case of the BLU estimator this is equivalent to minimizing the variance within $\mathrm{LU}$. We could now think of applying this Gauss-Markov approach also to our class of IE estimators. This would mean that we need to search for the minimum variance estimator within IEU, which we know is non-empty. Let us denote this estimator as $\hat{\theta}_{\text {BIEU }}$. Since we know that LU $\subset$ IEU $\subset$ IE, we must have the MSE ordering $\operatorname{MSE}\left(\hat{\theta}_{\mathrm{BIE}}\right) \leq \operatorname{MSE}\left(\hat{\theta}_{\mathrm{BIEU}}\right) \leq \operatorname{MSE}\left(\hat{\theta}_{\mathrm{BLU}}\right)$. It would therefore seem that from the standpoint of the MSE it is not advisable to use the minimum variance approach. But if we value the properties of unbiasedness and minimum variance, then $\theta_{\mathrm{BIEU}}$ would seem to be the proper contender for $\hat{\theta}_{\mathrm{BLU}}$. For IE estimation it turns out, however, that this difference is absent, since the two estimators $\hat{\theta}_{\mathrm{BIE}}$ and $\hat{\theta}_{\mathrm{BIEU}}$ can be shown to be identical. This is a direct consequence of the following GaussMarkov-like theorem.

Theorem 2: minimum variance unbiased estimation. The BIE estimator is unbiased and has an as good or better precision than the BLU-estimator:

1. $E\left\{\hat{\theta}_{\mathrm{BIE}}\right\}=E\left\{\hat{\theta}_{\mathrm{BLU}}\right\}$

2. $D\left\{\hat{\theta}_{\mathrm{BIE}}\right\} \leq D\left\{\hat{\theta}_{\mathrm{BLU}}\right\}$

where $D\{\cdot\}$ denotes the dispersion operator.

Proof of Theorem 2 is given in the appendix.

As the theorem shows, the BIE estimator is already unbiased by itself. Imposing the condition of unbiasedness onto the minimization problem of Eq. (13) would therefore not alter the solution. Hence $\hat{\theta}_{\mathrm{BIE}}=\hat{\theta}_{\mathrm{BIEU}}$.

The above result is remarkable since it shows that for a large class of PDFs of $y$, we can always, with a model like Eq. (1), improve upon the precision of the BLU estimator while keeping the estimator unbiased. If we apply the above theorem to the problem of estimating the baseline in case of GNSS and make the comparison with the 'float' baseline estimator and an unbiased 'fixed' baseline estimator, we have

$D\left\{\hat{b}_{\mathrm{BIE}}\right\} \leq D\{\check{b}\}$ and $E\left\{\hat{b}_{\mathrm{BIE}}\right\}=E\{\check{b}\}$

$D\left\{\hat{b}_{\mathrm{BIE}}\right\} \leq D\{\hat{b}\}$ and $E\left\{\hat{b}_{\mathrm{BIE}}\right\}=E\{\hat{b}\}$

The above matrix inequalities imply that $l^{T} D\left\{\hat{b}_{\mathrm{BIE}}\right\} l \leq l^{T} D\{\check{b}\} l$ and $l^{T} D\left\{\hat{b}_{\mathrm{BIE}}\right\} l \leq l^{T} D\left\{\hat{b}_{\mathrm{BLU}}\right\} l$ hold true for all $l \in R^{p}$. The precision of the baseline estimator $\hat{b}_{\text {BIE }}$ is therefore always better than or at least as good as the precision of its 'float' and 'fixed' counterparts.

We are now also in a position to make a comparison with our earlier result of Eq. (8). It will be clear that the performance statement of Eq. (19) is weaker than that of Eq. (8). On the other hand, Eq. (8) is only valid in the case that the probability of correct integer estimation is sufficiently close to one, whereas the above result is always valid.

We know from standard adjustment theory that the BLU residuals are uncorrelated with the BLU estimators. This is a consequence of the minimum variance property of the BLU estimator. The following lemma shows that this property is inherited by the BIE estimator as well.

Lemma 2: zero correlation. Let the BIE residual be defined as $\hat{e}_{\mathrm{BIE}}=\theta_{\mathrm{IE}}-\hat{\theta}_{\mathrm{BIE}}$, with $\theta_{\mathrm{IE}}$ an arbitrary $I E$ estimator, and let $\sigma_{\hat{\theta}_{\mathrm{BIE}} \hat{e}_{\mathrm{BIE}}}$ denote the covariance between $\hat{\theta}_{\mathrm{BIE}}$ and $\hat{e}_{\mathrm{BIE}}$. Then

$\sigma_{\hat{\theta}_{\mathrm{BIE}} \hat{e}_{\mathrm{BIE}}}=0$

Proof of Lemma 2 is given in the appendix.

Note that since $\hat{\theta}_{\mathrm{BLU}}$ is an IE estimator as well, $\hat{\theta}_{\mathrm{BIE}}$ and $\hat{\theta}_{\mathrm{BLU}}-\hat{\theta}_{\mathrm{BIE}}$ are also uncorrelated.

\subsection{The Gaussian case}

In our derivation of the BIE estimator and its properties we have not so far made a particular choice for the PDF of $y$. In many applications, however, for example GNSS, it is often assumed that $y$ is normally distributed. In that case the PDF of $y$ takes the form

$p_{y}(y)=\frac{1}{(2 \pi)^{\frac{m}{2}} \sqrt{\operatorname{det} Q_{y}}} \exp \left\{-\frac{1}{2}\|y-A a-B b\|_{Q_{y}}^{2}\right\}$

where $\|\cdot\|_{Q_{v}}^{2}=(\cdot)^{T} Q_{y}^{-1}(\cdot)$. With this Gaussian PDF the BIE estimator also takes on a particular shape. We have the following corollary.

Corollary: BIE in the Gaussian case. Let the PDF of $y$ be given as in Eq. (21) and let $\hat{\theta}_{\mathrm{BIE}}$ be the BIE estimator of $\theta=l_{a}^{T} a+l_{b}^{T} b$. Then 
$\hat{\theta}_{\mathrm{BIE}}=l_{a}^{T} \hat{a}_{\mathrm{BIE}}+l_{b}^{T} \hat{b}_{\mathrm{BIE}}$

with

$\hat{a}_{\mathrm{BIE}}=\sum_{z \in Z^{n}} z \frac{\exp \left\{-\frac{1}{2}\|\hat{a}-z\|_{Q_{\hat{a}}}^{2}\right\}}{\sum_{z \in Z^{n}} \exp \left\{-\frac{1}{2}\|\hat{a}-z\|_{Q_{\hat{a}}}^{2}\right\}}$

$\hat{b}_{\mathrm{BIE}}=\hat{b}-Q_{\hat{b} \hat{a}} Q_{\hat{a}}^{-1}\left(\hat{a}-\hat{a}_{\mathrm{BIE}}\right)$

Proof of the Corollary is given in the appendix.

This result shows that in the Gaussian case we may use the three-step procedure of Sect. 2.2 also for BIE estimation. The only difference is that we need to replace the integer estimator $\check{a}$ by the BIE estimator $\hat{a}_{\text {BIE }}$.

Since the space of integers $Z^{n}$ can be seen as a certain discretized version of the space of real numbers $R^{n}$, we would expect that if the integer grid size gets smaller in relation to the size and extent of the PDF, the difference between the two estimators $\hat{a}_{\mathrm{BIE}}$ and $\hat{a}$ would get smaller as well. Similarly, if the PDF gets more peaked in relation to the integer grid size, we would expect that the BIE estimator $\hat{a}_{\mathrm{BIE}}$ would tend to an integer estimator. This is made precise in the following lemma.

Lemma 3: limits of the integer grid.

1. If we replace $\sum_{z \in Z^{n}}$ by $\int_{R^{n}} d z$ in Eq. (22), then

$$
\hat{a}_{\mathrm{BIE}}=\hat{a}
$$

2. Let the vc matrix of $\hat{a}$ be factored as $Q_{\hat{a}}=\sigma^{2} G$. Then

$$
\lim _{\sigma \rightarrow 0} \hat{a}_{\mathrm{BIE}}=\check{a}_{\mathrm{ILS}}
$$

Proof of Lemma 3 is given in the appendix.

It is interesting to observe that the above expressions given for $\hat{a}_{\mathrm{BIE}}$ and $\hat{b}_{\mathrm{BIE}}$ are identical to their Bayesian counterparts as given in Betti et al. (1993) and Gundlich and Koch (2002); see also Teunissen (2001) and Gundlich and Teunissen (2003). This is not quite true for the general case. However, the above equivalence nicely bridges the gap which has existed so far between the current theory of integer inference and the Bayesian approach. Despite the similarity in the above case, however, there are important differences in the probabilistic evaluation of the solutions. Like the BLU estimator, the BIE estimator is a random variable with the property of being unbiased and of minimum variance. In the Bayesian framework the solution is considered to be non-random due to the conditioning that takes place. Furthermore, in the Bayesian framework the unknown parameters are assumed to be random variables for which probability distributions need to be specified a priori. The theory developed in the present contribution is non-Bayesian throughout, with no need at all to make assumptions about prior distributions.

\section{Summary}

This study was motivated by the problem of GNSS carrier phase ambiguity resolution. We know that when the ambiguity success rate of the usual GNSS model is large enough, the 'fixed' baseline estimator outperforms its 'float' counterpart in the sense that

$P\left(\check{b} \in E_{\mathrm{B}}\right) \geq P\left(\hat{b} \in E_{\mathrm{B}}\right)$

for any convex region $E_{b} \subset R^{p}$ symmetric with respect to the unknown but true baseline $b \in R^{p}$. This is a strong result, but unfortunately only valid if the probability of correct integer estimation is sufficiently close to one. We therefore raised the question whether it would be possible to devise a baseline estimator which always outperforms its 'float' counterpart. Our approach to this problem was to opt for a weaker performance criterion and a larger class of estimators to choose from. As performance criterion we chose to use the MSE.

The new class of estimators that we introduced we referred to as the class of integer equivariant estimators, IE. This class is larger than the class of integer estimators, but it still obeys the integer remove-restore principle. In order to devise our own IE estimator, a useful representation of the IE estimators was given. We then presented the BIE estimator of an arbitrary linear function of the integer as well as real-valued parameters of the GNSS model. This was done by solving the problem of minimizing the MSE within the IE class. The expression given for the BIE estimator holds true for any PDF the data might have.

It was also shown that the class of linear unbiased estimators, LU, is a subset of the class of IE estimators, LU $\subset$ IE. This automatically implies that, in the MSE sense, the BIE estimator always outperforms its BLU counterpart. In addition it was shown that the BIE estimator is unbiased. Hence, similar to the well-known Gauss-Markov theorem, which states that the minimum variance unbiased estimator within the class of linear estimators is given by the LS estimator BLU, we obtained a Gauss-Markov-like theorem stating that the minimum variance unbiased estimator within the IE class is given by the LMS estimator BIE. Both theorems hold true for any PDF the data might have. For the BLU solution we need to know the vc matrix of the data up to a proportionality constant, whereas for the BIE solution we need to know the PDF up to a proportionality factor.

When the theory is applied to solve for the baseline vector in the GNSS model, we have as a direct consequence of the theory that

$$
\operatorname{MSE}\left(\hat{b}_{\mathrm{BIE}}\right) \leq \operatorname{MSE}(\hat{b}) \text { and } \operatorname{MSE}\left(\hat{b}_{\mathrm{BIE}}\right) \leq \operatorname{MSE}(\check{b})
$$

Hence the MSE of the BIE estimator of the baseline is less than or at the most equal to, that of both its 'float' and 'fixed' counterparts. Although this is a much weaker performance statement than Eq. (23), it has the advantage of being valid all the time. 
Acknowledgements. This contribution was finalized during the author's stay, as a Tan Chin Tuan Professor, at the Nanyang Technological University's GPS Centre (GPSC) in Singapore. The hospitality of the GPSC's director Prof Law Choi Look and his colleagues is greatly appreciated.

\section{Appendix}

Proof of Lemma 1. We will start with the 'if' part: If $g_{\theta}(\alpha, \beta, \gamma)=l_{a}^{T} \alpha+l_{b}^{T} \beta+h_{\theta}(\alpha, \gamma)$ with $h_{\theta}$ periodic in its first slot, then clearly $g_{\theta}(\alpha+z, \beta+\zeta, \gamma)=$ $g_{\theta}(\alpha, \beta, \gamma)+l_{a}^{T} z+l_{b}^{T} \zeta, \forall z \in Z^{n}, \zeta \in R^{p}$. Therefore, since $f_{\theta}(A \alpha+B \beta+C \gamma)=g_{\theta}(\alpha, \beta, \gamma)$ and $y=A \alpha+B \beta+C \gamma$ with $(A, B, C)$ invertible

$$
\begin{aligned}
f_{\theta}(y+A z+B \zeta)= & f_{\theta}(y)+l_{a}^{T} z+l_{b}^{T} \zeta, \\
& \forall y \in R^{m}, z \in Z^{n}, \zeta \in R^{p}
\end{aligned}
$$

which is equivalent to the properties of Definition 2.

For the 'only if' part we have: if Eq. (A1) holds true, the $h_{\theta}$ function defined as $h_{\theta}(\alpha, \gamma)=f_{\theta}(A \alpha+B \beta+C \gamma)-$ $l_{a}^{T} \alpha-l_{\mathrm{B}}^{T} \beta$ with $(A, B, C)$ invertible will be periodic in its first slot and hence $g_{\theta}(\alpha, \beta, \gamma)=f_{\theta}(A \alpha+B \beta+C \gamma)$ can be written as $g_{\theta}(\alpha, \beta, \gamma)=l_{a}^{T} \alpha+l_{\mathrm{B}}^{T} \beta+h_{\theta}(\alpha, \gamma)$.

Proof of Theorem 1 Let $\hat{\theta}_{\mathrm{IE}}=f_{\theta}(y)$. Then $\operatorname{MSE}\left(\hat{\theta}_{\mathrm{IE}}\right)=$ $E\left\{\left(f_{\theta}(y)-\theta\right)^{2}\right\}$ needs to be minimized for all functions $f_{\theta} \in$ IE. We first apply the change of variables $y=A \alpha+B \beta+C \gamma$ with $(A, B, C)$ invertible, together with $\theta=l_{a}^{T} a+l_{b}^{T} b$ and our representation $f_{\theta}(A \alpha+$ $B \beta+C \gamma)=l_{a}^{T} \alpha+l_{b}^{T} \beta+h_{\theta}(\alpha, \gamma)$. This gives $\operatorname{MSE}\left(\hat{\theta}_{\mathrm{IE}}\right)=$ $E\left\{\left[l_{a}^{T}(\alpha-a)+l_{b}^{T}(\beta-b)\right]^{2}\right\}+H\left(h_{\theta}\right)$ with

$H\left(h_{\theta}\right)=E\left\{2\left[l_{a}^{T}(\alpha-a)+l_{b}^{T}(\beta-b)\right] h_{\theta}(\alpha, \gamma)+h_{\theta}(\alpha, \gamma)^{2}\right\}$

Hence we may now concentrate on minimizing $H\left(h_{\theta}\right)$. Using the expectation property $E\{\cdot\}=$ $E_{\gamma}\left\{E_{\alpha}\{\cdot \mid \alpha, \gamma\} \mid \gamma\right\}$, we may write for $H$

$$
\begin{aligned}
H\left(h_{\theta}\right)= & E_{\gamma}\left\{E _ { \alpha } \left\{2\left[l_{a}^{T}(\alpha-a)+l_{b}^{T} E_{\beta}\{\beta-b \mid \alpha, \gamma\}\right] h_{\theta}(\alpha, \gamma)\right.\right. \\
& \left.\left.+h_{\theta}(\alpha, \gamma)^{2} \mid \gamma\right\}\right\}
\end{aligned}
$$

If we now can find an admissible $h_{\theta}$ which minimizes the inner expectation $E_{\alpha}\{\cdot \mid \gamma\}$ for all $\gamma$, then also $H\left(h_{\theta}\right)$ would be minimized. Using $h_{\theta}(\alpha+z, \gamma)=h_{\theta}(\alpha, \gamma)$ and the fact that $\int f(x) \mathrm{d} x=\int_{S_{0}} \sum_{z \in Z^{n}} f(x+z) \mathrm{d} x$ holds true for any pull-in region $S_{0}$ and any $f$, we may write

$$
\begin{gathered}
H\left(h_{\theta}\right)=E_{\gamma}\left\{\int_{S_{0}}\left[\left(h_{\theta}(\alpha, \gamma)-\hat{h}_{\theta}(\alpha, \gamma)\right)^{2}-\left(\hat{h}_{\theta}(\alpha, \gamma)\right)^{2}\right]\right. \\
\left.\quad \times \sum_{z \in Z^{n}} p_{\alpha \mid \gamma}(\alpha+z \mid \gamma) \mathrm{d} \alpha \mid \gamma\right\}
\end{gathered}
$$

with $p_{\alpha \mid \gamma}(\alpha \mid \gamma)$ the PDF of $\alpha$ given $\gamma$ and with

$$
\hat{h}_{\theta}(\alpha, \gamma)=\frac{\sum_{z \in Z^{n}} \int\left[l_{a}^{T}(a-z-\alpha)+l_{b}^{T}(b-\beta)\right] p_{\alpha, \beta, \gamma}(\alpha+z, \beta, \gamma) \mathrm{d} \beta}{\sum_{z \in Z^{n}} \int p_{\alpha, \beta, \gamma}(\alpha+z, \beta, \gamma) \mathrm{d} \beta}
$$

This shows that $h_{\theta}(\alpha, \gamma)=\hat{h}_{\theta}(\alpha, \gamma)$ is the sought-for minimum. We therefore have, with $\hat{f}(A \alpha+B \beta+C \gamma)=l_{a}^{T} \alpha+l_{b}^{T} \beta+\hat{h}_{\theta}(\alpha, \gamma)$, that

$$
\begin{aligned}
& \hat{f}(A \alpha+B \beta+C \gamma) \\
& =\frac{\sum_{z \in Z^{n}} \int\left[l_{a}^{T} z+l_{b}^{T} \bar{\beta}\right] p_{\alpha, \beta, \gamma}(\alpha+a-z, \beta+b-\bar{\beta}, \gamma) \mathrm{d} \bar{\beta}}{\sum_{z \in Z^{n}} \int p_{\alpha, \beta, \gamma}(\alpha+z, \bar{\beta}, \gamma) \mathrm{d} \bar{\beta}}
\end{aligned}
$$

Since $y=A \alpha+B \beta+C \gamma$ with $(A, B, C)$ invertible and $p_{\alpha, \beta, \gamma}(\alpha, \beta, \gamma)=\operatorname{det}(A, B, C) p_{y}(A \alpha+B \beta+C \gamma)$, the result follows.

Proof of Theorem 2. With the result of Theorem 1, Theorem 2 is proven once it can be shown that $\hat{\theta}_{\mathrm{BIE}}$ is unbiased. Using the IE representation $\hat{\theta}_{\mathrm{BIE}}=$ $l_{a}^{T} \alpha+l_{b}^{T} \beta+\hat{h}_{\theta}(\alpha, \gamma)$ where $y=A \alpha+B \beta+C \gamma$, we first note that $E\{\alpha\}=a$ and $E\{\beta\}=b$ for any matrix $C$ for which $(A, B, C)$ is invertible. Therefore $E\left\{\hat{\theta}_{\mathrm{BIE}}\right\}=\theta$ if and only if $E\left\{\hat{h}_{\theta}(\alpha, \gamma)\right\}=0$. This is easily verified using Eq. (A2).

Proof of Lemma 2 First note that $\hat{\theta}_{\mathrm{IE}}=\hat{\theta}_{\mathrm{BIE}}+$ $\lambda\left(\hat{\theta}_{\mathrm{BLU}}-\hat{\theta}_{\mathrm{BIE}}\right)$ is an IE estimator for any $\lambda \in R$. Application of the error propagation law gives $\sigma_{\hat{\theta}_{1 F}}^{2}=$ $\sigma_{\hat{\theta}_{\mathrm{BIE}}}^{2}+2 \lambda \sigma_{\hat{\theta}_{\mathrm{BIE}}\left(\hat{\theta}_{\mathrm{BLU}}-\hat{\theta}_{\mathrm{BIE}}\right)}+\lambda^{2} \sigma_{\left(\hat{\theta}_{\mathrm{BLU}}-\hat{\theta}_{\mathrm{BIE}}\right)}^{2}$. Since $\sigma_{\hat{\theta}_{\mathrm{BIE}}}^{2} \leq \sigma_{\hat{\theta}_{\mathrm{II}}}^{2}$, we must have that $2 \lambda \sigma_{\hat{\theta}_{\mathrm{BIE}}\left(\hat{\theta}_{\mathrm{BLU}}-\hat{\theta}_{\mathrm{BIE}}\right)}+\lambda^{2} \sigma_{\left(\hat{\theta}_{\mathrm{BLU}}-\hat{\theta}_{\mathrm{BIE}}\right)} \geq 0$, $\forall \lambda \in R$. This is only possible if $\sigma_{\hat{\theta}_{\mathrm{BIE}}\left(\hat{\theta}_{\mathrm{BLU}}-\hat{\theta}_{\mathrm{BIE}}\right)}=0$. Note that the result also holds when $\hat{\theta}_{\mathrm{BLU}}$ is replaced by an arbitrary IE estimator.

Proof of Corollary. The result Eq. (A3) holds true for any $C$ for which $(A, B, C)$ is invertible. Let us assume that matrix $C$ can be chosen such that $\alpha$ and $\beta$ become independent of $\gamma$. Then according to Eq. (A3), the dependence on $\gamma$ disappears and $\hat{a}_{\mathrm{BIE}}$ and $\hat{b}_{\mathrm{BIE}}$ can be written as

$$
\begin{gathered}
\hat{a}_{\mathrm{BIE}}=\sum_{z \in Z^{n}} z \frac{p_{\alpha}(\alpha+a-z)}{\sum_{z \in Z^{n}} p_{\alpha}(\alpha+a-z)} \\
\hat{b}_{\mathrm{BIE}}=\beta-\sum_{z \in Z^{n}} E\{\beta-b \mid \alpha+a-z\} \\
\times \frac{p_{\alpha}(\alpha+a-z)}{\sum_{z \in Z^{n}} p_{\alpha}(\alpha+a-z)}
\end{gathered}
$$

If we choose $C$ such that $C^{T} Q_{y}^{-1}(A, B)=0$, then $\alpha=\hat{a}$, $\beta=\hat{b}$ and $C \gamma=\hat{e}$, with the $\operatorname{LS}$ residual $\hat{e}=y-A \hat{a}-B \hat{b}$. If we further assume that $y$ is normally distributed, then $\hat{e}$, and thus $\gamma$, become independent of $\alpha=\hat{a}$ and $\beta=\hat{b}$. The PDF of $\alpha=\hat{a}$ and the conditional mean $E\{\beta \mid \alpha+a-z\}$ then reduce to

$$
\begin{aligned}
& p_{\hat{a}}(x)=\frac{1}{(2 \pi)^{\frac{n}{2}} \sqrt{\operatorname{det} Q_{\hat{a}}}} \exp \left\{-\frac{1}{2}\|x-a\|_{Q_{\hat{a}}}^{2}\right\} \\
& E\{\beta \mid \alpha+a-z\}=b-Q_{\hat{b} \hat{a}} Q_{\hat{a}}^{-1}(z-\hat{a})
\end{aligned}
$$

Substitution into Eq. (A4) proves the result. 


\section{Proof of Lemma 3.}

1. Since $\int x \exp \left\{-\frac{1}{2}\|x-\hat{a}\|_{Q_{\hat{a}}}^{2}\right\} \mathrm{d} x=\hat{a}(2 \pi)^{\frac{n}{2}} \sqrt{\operatorname{det} Q_{\hat{a}}}$ and $\int \exp \left\{-\frac{1}{2}\|x-\hat{a}\|_{Q_{\hat{a}}}^{2}\right\} \mathrm{d} x=(2 \pi)^{\frac{n}{2}} \sqrt{\operatorname{det} Q_{\hat{a}}}$, the result follows.

2. Let $S_{u}=\left\{x \in R^{n} \mid\|x-u\|_{Q_{a}}^{2} \leq\|x-z\|_{Q_{\hat{a}}}^{2}, \forall z \in Z^{n}\right\}$ be the ILS pull-in region of $u \in Z^{n}$. Then $S_{u}$ will be independent of $\sigma$ if we factor the vc matrix of $\hat{a}$ as $Q_{\hat{a}}=\sigma^{2} G$. From

$$
\begin{aligned}
w_{u}(x) & =\frac{\exp \left\{-\frac{1}{2}\|x-u\|_{Q_{a}}^{2}\right\}}{\sum_{z \in Z^{n}} \exp \left\{-\frac{1}{2}\|x-z\|_{Q_{\hat{a}}}^{2}\right\}} \\
& =\frac{1}{1+\sum_{z \neq u} \exp \left\{-\frac{1}{2} \sigma^{-2}\left\{\|x-z\|_{G}^{2}-\|x-u\|_{G}^{2}\right\}\right\}}
\end{aligned}
$$

it then follows that $\lim _{\sigma \mapsto 0} w_{u}(x)=1$ and $\lim _{\sigma \mapsto 0} w_{z}(x)=0, \forall z \neq u$, if $x \in S_{u}$. The last equation is a consequence of $\sum_{z \in Z^{n}} w_{z}(x)=1, \forall x \in R^{n}$. Since $w_{z}(x)$ reduces in the limit to the indicator function of the ILS pull-in region, the result follows.

\section{References}

Betti B, Crespi M, Sanso F (1993) A geometric illustration of ambiguity resolution in GPS theory and a Bayesian approach. Manuscr Geod 18:317-330

Gundlich B, Koch K-R (2002) Confidence regions for GPS baselines by Bayesian statistics. J Geod 76: 55-62
Gundlich B, Teunissen PJG (2003) Multiple models fixed, switching and interacting. Proc V Hotine-Marussi Symposium, Matera, Italy, in press

Hofmann-Wellenhof B, Lichtenegger H, Collins J (2001) Global positioning system: theory and practice, 5th edn. Springer, Berlin Heidelberg New York

Leick A (1995) GPS satellite surveying. 2nd edn. John Wiley, New York

Misra P, Enge P (2001) Global positioning system: signals, measurements, and performance. Ganga-Jamuna Press

Parkinson B, Spilker JJ (eds) (1996) GPS: theory and applications, vols 1 and 2. AIAA, Washington, DC

Strang G, Borre K (1997) Linear algebra, geodesy, and GPS. Wellesley-Cambridge Press

Teunissen PJG (1993) Least-squares estimation of the integer GPS ambiguities. Invited Lecture, Section IV Theory and Methodology, IAG General Meeting, Beijing, China, August. Also in: LGR Series, no. 6, Delft Geodetic Computing Centre, Delft

Teunissen PJG (1995) The least-squares ambiguity decorrelation adjustment: a method for fast GPS integer ambiguity estimation. J Geod 70:65-82

Teunissen PJG (1999a) The probability distribution of the GPS baseline for a class of integer ambiguity estimators. J Geod 73:275-284

Teunissen PJG (1999b) An optimality property of the integer leastsquares estimator. J Geod 73:587-593

Teunissen PJG (2001) Statistical GNSS carrier phase ambiguity resolution: a review. Proc. IEEE Symp Statistical Signal Processing, Singapore, pp 4-12

Teunissen PJG, Kleusberg A (eds) (1998) GPS for geodesy, 2nd edn. Springer, Berlin Heidelberg New York 